\title{
La percepción laboral de los egresados del modelo de la universidad intercultural en México, el caso de la UNICH y la UIEM
}

\section{Occupational perception of the alumni of the Intercultural University model in Mexico, situation at the UNICH and the UIEM}

\author{
Zuzana Erdösová \\ Universidad Autónoma del Estado de México, México \\ zerdosova@uaemex.mx \\ https://orcid.org/0000-0001-7924-7599 \\ Rafael Juárez-Toledo \\ Universidad Autónoma del Estado de México, México \\ rjuarezt@uaemex.mx \\ https://orcid.org/0000-0003-3667-5125
}

(Recibido: 07/04/2020; Aceptado: 10/04/2020; Versión final recibida: 15/05/2020)

Cita del artículo: Erdösová Z. y Juárez-Toledo R. (2020). La percepción laboral de los egresados del modelo de la universidad intercultural en México, el caso de la UNICH y la UIEM. Revista Cátedra, 3(2), 35-51.

\section{Resumen}

Los licenciados interculturales son jóvenes procedentes de variados ámbitos socioculturales que se forman en el marco del modelo educativo llamado universidad intercultural, gestionado por la Secretaría de Educación Pública de México. En este trabajo se analiza la disyuntiva existente entre el discurso interculturalista plasmado en los perfiles de ingreso y egreso que soportan a la educación superior de este tipo, y la situación imperante para sus egresados toda vez que buscan insertarse en el mercado laboral. El cuestionamiento inicial al que se hace referencia para ubicar el problema es: ¿qué sucede una vez concluida la formación universitaria intercultural? Aunque la percepción sobre la educación intercultural es positiva para sus autoridades y egresados, se ha identificado una

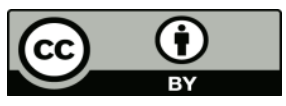

Licencia Creative Commons Atribución 4.0 Internacional (CC BY 4.0) 
profunda incomprensión o desconocimiento de la propuesta intercultural y del papel que los licenciados interculturales deben desempeñar en la sociedad, específicamente en el mercado de trabajo. Los egresados deben enfrentar problemas relacionados con la discriminación, los bajos niveles salariales y la falta de eficientes oportunidades de desarrollo personal. Mediante un proceso cualitativo, aplicado en los diferentes actores, se ha obtenido la percepción que se tiene sobre el desempeño de los egresados interculturales para dos contextos geográficos y socioculturales: la Universidad Intercultural del Estado de México y la Universidad Intercultural de Chiapas.

\section{Palabras clave}

Educación intercultural, egresado intercultural, mercado laboral, Universidad intercultural, UNICH, UIEM

\section{Abstract}

The intercultural bachelor is a term that refers to the young people from diverse sociocultural environments who took their degree in the framework of the recently founded educational model called Intercultural University, administered by the Mexican Ministry of Education. In this paper we analyze the uneasy position of intercultural discourse incorporated in the admission and graduation profiles, which give support to superior education, and the real situation in the labor market. The initial question, and key issue, is: what happens after the completion of the intercultural university education? The perception about of this Intercultural discourse is positive from authorities and students, but the situation outside the Intercultural Universities (above all in the labor market) which is marked by a deep miscomprehension or ignorance of given of the role that the intercultural bachelors should carry in the society. They have many problems as discrimination, low salary levels and inefficient programs of individual development. Through a qualitative process we obtain the perception from several actors about of the role of the intercultural bachelors. The research was carried out in two geographical and sociocultural contexts, the states of México: Intercultural University of State of Mexico and Intercultural University of Chiapas.

\section{Keywords}

Intercultural university, intercultural education, intercultural bachelor, labor market, UNICH, UIEM

\section{Introducción}

Los cambios estructurales por los que ha atravesado México y que son reflejo de las constantes reformas constitucionales, con vías a consolidar la apertura, la privatización y la desregulación, han provocado la presión económica y social en los contextos locales. No está oculto que para ciertos grupos de población el desempeño laboral atiende rasgos de marginación y precariedad, tal es el caso de la población indígena. Al respecto, los grupos étnicos han tenido que participar en fuertes oleadas migratorias (Cárdenas, 2014, Granados y Quezada, 2018) o han tenido que ajustar sus estilos de vida dentro de sus propias localidades con vías a elevar sus niveles de competitividad y lograr, con ello, obtener los recursos económicos para la subsistencia. Ya sea en el contexto urbano o en el rural, la población indígena aparece con las mayores tasas de marginación, pobreza y exclusión dentro del país como lo presentan diversos documentos y comunicados oficiales (Coneval, 2018a; CDI, 2018; INEGI, 2018; INEGI 2020). 
De manera específica, en México, se perciben con claridad las brechas educativas que colocan a la población de los pueblos originarios en fuertes desventajas. Acorde con los datos presentados por el INPI (2010 y 2017) el promedio de escolaridad para México, en 2005 , fue de 8,8 años, pero la población indígena se ubicó por debajo de este indicador con 7.1 años. Con datos proyectados para 2015, se observa que el panorama escolar no ha cambiado y que en todos los niveles de instrucción formal (salvo el nivel primario) es la población indígena la menos favorecida. Por ejemplo, en el grupo de población sin algún tipo de instrucción la población indígena se ubicó en 16.6 \% (la media nacional fue de $6 \%$ ); a nivel de estudios de primaria completa la población indígena sostuvo una cobertura de $18.2 \%$ (la media nacional fue de $15 \%$ ), pero conforme se avanza en los niveles se intensifica la problemática. Así para el nivel secundaria la población indígena mostró acceso en $20.5 \%$ (la media nacional fue de $23.7 \%$ ); y para los niveles media superior (bachillerato) y superior el nivel de incorporación fue de 21.7 \% para la población indígena (la media nacional fue de $40.7 \%$ ).

Ante este panorama, desde diferentes instancias públicas se han estructurado diversos programas educativos que llevan la finalidad de servir como un canal para equilibrar las desigualdades entre la población mexicana, en particular para la población que representa a los pueblos originarios.

Uno de los retos en el diseño del nuevo modelo educativo y del conjunto de la acción pública es promover mejores resultados para todos los estudiantes $\mathrm{y}$, al mismo tiempo, cerrar las brechas educativas que históricamente han prevalecido entre distintos sectores escolares. Esta doble tarea, sin embargo, exige apego al principio de equidad para que la educación favorezca la igualdad social (INEE, 2017a, pág. 7).

Estos programas han ajustado sus propuestas de acuerdo con el sistema educativo del que se trate, aunque en su mayoría asumen a dicha población como un grupo de atención especial, o en condición vulnerable, ante lo cual la atención generalmente es de tipo asistencialista. Por ejemplo, en el documento ejecutivo sobre política educativa del Instituto Nacional para la Evaluación Educativa (INEE, 2017b) se hace mención a 29 programas que se vinculan con la educación indígena básica, cuya justificación se asienta en el derecho a la educación y como una retribución a la deuda histórica respecto a los pueblos indígenas.

Lo anterior, aunado a la importante heterogeneidad y vulnerabilidad social y económica de dicha población, hacen necesaria la construcción de acciones afirmativas dirigidas a revertir las brechas a partir de políticas inclusivas, transversales y que respeten las particularidades de la niñez indígena, para terminar con una larga historia de discriminación, racismo y exclusión (INEE, 2017a, pág. 2).

Con respecto a la educación de tipo superior, la educación intercultural universitaria surgió en México con la intención discursiva de promover la inclusión de las poblaciones indígenas en los ámbitos del desarrollo nacional. Al tiempo que fuera posible crear un ambiente más justo y equitativo, no sólo en el acceso a la educación superior, también para crear un tipo de profesionista con capacidades para intermediar entre sus comunidades de origen y el entorno. Sin embargo, los rasgos y los elementos conceptuales con los que fue integrada, lo cual se puede percibir en la visión, misión y perfiles de ingreso y egreso, se podrían considerar no acordes con la realidad. Vázquez-Parra, J. y Campos-Rivas, C. (2016) destacan el hecho de que:

Licencia Creative Commons Atribución 4.0 Internacional (CC BY 4.0)

Revista Cátedra, 3(2), pp. 35-51, mayo-agosto 2020. e-ISSN: 2631-2875

https://doi.org/10.29166/10.29166/catedra.v3i2.2188 
aunque en las últimas décadas han crecido los programas públicos para equilibrar la posición educativa y laboral de la población indígena, en la práctica dicha población debe asumir los problemas estructurales del desempleo, aunado a vicios y prácticas discriminatorias que se observan en la selección de puestos y en la asignación de las remuneraciones" (p. 830).

Esta idea da muestra de que desde la educación intercultural se ha estado atendiendo un lado del problema para las poblaciones originarias, al ubicar una serie de universidades en lugares estratégicos para lograr el mayor acceso y ampliar la cobertura educativa. Pero ¿qué sucede con los egresados una vez concluida la formación universitaria intercultural? Ha resultado, además de interesante, necesario realizar un acercamiento a las instituciones educativas interculturales, para observar y documentar los problemas a los que se están enfrentando las recientes generaciones de egresados. Toda vez que no existen, por el momento, los programas para su incorporación al campo laboral de manera directa y ventajosa; de manera que hay una incongruencia entre el perfil de formación y la posterior carencia de puestos de trabajo. Así, este grupo de profesionistas debe competir frente a otros que fueron formados en otras opciones universitarias en un mercado de trabajo que los desconoce.

Así, los principales cuestionamientos que fueron planteados para dar curso a la investigación, cuyos resultados aquí se presentan, son los siguientes: respecto al modelo educativo de la universidad intercultural ¿ha sido capaz de plasmarse en una formación sólida de egresados "de otro tipo" que consecuentemente actúen en la sociedad según los valores interculturales?, ¿cuántos egresados realmente han logrado establecerse en áreas profesionales que están en sintonía con su formación intercultural?, ¿qué tan probable es que los exalumnos (sobre todo los que declaran tener una vocación intercultural) podrán practicar plenamente dicha vocación fuera de sus instituciones de formación?, ¿cómo se perciben los egresados respecto a sus competencias de formación, con relación a las oportunidades que se ofrecen en los mercados de trabajo actuales?

El propósito del artículo es presentar la discrepancia existente entre el discurso interculturalista "políticamente correcto" que, a pesar de sus deficiencias, en muchos casos logra transformar positivamente las opiniones de los alumnos respecto a la diversidad cultural mexicana; y la situación imperante fuera de los ámbitos académicos de las Universidades Interculturales, específicamente en el mercado laboral. Se han considerado dos casos representativos: la Universidad Intercultural del Estado de México (UIEM) y la Universidad Intercultural de Chiapas (UNICH).

El abordaje metodológico se realizó con un trabajo de campo orientado a extraer la percepción que se tiene sobre la valoración del licenciado intercultural desde la opinión de los egresados y de las autoridades de ambas instituciones. Lo anterior se hizo en referencia a los perfiles de ingreso y egreso, y la práctica profesional de sus egresados.

El artículo presenta, inicialmente, el enfoque que ha atendido la educación superior intercultural en México, a fin de hacer notar los rasgos fundamentales desde los que se basa el discurso intercultural, mismo que permea entre sus actores. Como segundo elemento, se dan a conocer los pormenores metodológicos a través de los cuales se diseñó y aplicó una guía de entrevista en jóvenes recién egresados de la UNICH y de la UIEM, y se indica cómo se evaluó la relación de congruencia entre el perfil de egreso (PE) y el tipo de trabajo (T) en el que se desempeñaba la persona al momento de la entrevista. Finalmente, en la parte

Licencia Creative Commons Atribución 4.0 Internacional (CC BY 4.0)

Revista Cátedra, 3(2), pp. 35-51, mayo-agosto 2020. e-ISSN: 2631-2875

https://doi.org/10.29166/10.29166/catedra.v3i2.2188 
dedicada a los resultados se presentan las diferentes versiones de opinión, o percepción, desde las autoridades de las dos universidades, así como de los egresados que constituyeron la muestra, para interpretar el grado de éxito que hasta el momento ha tenido la Universidad Intercultural respecto a la inserción de sus licenciados interculturales y las metas, por demás ambiciosas, que deben atender a diferencia de los profesionistas convencionales.

\section{La universidad intercultural}

La interculturalidad refiere a un discurso que busca reconocer la existencia de contacto y de relaciones entre culturas diferentes. Si bien su construcción conceptual pudiera ser motivo de amplio debate, se puede distinguir la aceptación de su base ética que engloba la idea de respeto y diálogo. En materia educativa, los programas de estudio basados en la interculturalidad llevan el propósito de apoyar el reconocimiento de la diversidad cultural existente, tal es el caso de México; país donde se reconoce de manera constitucional la pluralidad basada en sus grupos étnicos, que en conjunto corresponde al $10.1 \%$ de la población total (INPI, 2017).

A diferencia de otros casos en América Latina en los cuales los participantes de los pueblos originarios han sido la base para la promoción del modelo educativo intercultural, en México el modelo tuvo un origen institucionalizado bajo el auspicio de la Secretaría de Educación Pública (SEP). Dicho modelo educativo surgió en el año 2000 y actualmente se ha esparcido a través de 11 Universidades dentro de las siguientes entidades federativas: Tabasco, Michoacán, Chiapas, Estado de México, Puebla, Guerrero, Veracruz, Quintana Roo, Hidalgo, Sinaloa, San Luís Potosí. El modelo entero dispone de una oferta de 32 licenciaturas, seis maestrías y cuatro doctorados (SEP, 2018).

La universidad intercultural en México se estructuró en apego a los principios fundamentales de la Educación Intercultural que emanan desde la UNESCO (2006) ${ }^{1}$. La incorporación de los principios de la educación intercultural al ámbito de la educación universitaria dio como resultado el diseño y la oferta de una diversidad de carreras con perfiles diferenciados, acorde con el entorno específico para cada Universidad. No obstante, a lo anterior se observa que, en lo general, tanto los perfiles de ingreso como los perfiles de egreso buscan culminar con la preparación de un tipo de profesionista que constituya un puente para la organización, difusión y promoción de aquellos espacios donde radica la fuerza cultural comunitaria o indígena (SEP, 2018).

Sin embargo, estos principios permiten entender la relevancia por adoptar ambientes educativos dirigidos hacia el respeto y la tolerancia, al mismo tiempo que se permita la capacidad de elevar los niveles de competitividad educativa y laboral sin importar la condición étnica o cultural de sus practicantes, pero en la realidad sucede una distorsión de sus componentes. Erdösová (2013) había sugerido que la universidad intercultural, si bien

${ }^{1}$ La educación intercultural respeta la identidad cultural del educando impartiendo a todos una educación de calidad que se adecúe y adapte a su cultura. 2. La educación intercultural enseña a cada educando los conocimientos, las actitudes y las competencias culturales necesarias para que pueda participar plena y activamente en la sociedad. 3. La educación intercultural enseña a todos los educandos los conocimientos, actitudes y las competencias culturales que les permiten contribuir al respeto, el entendimiento y la solidaridad entre individuos, entre grupos étnicos, sociales, culturales y religiosos y entre naciones.

Licencia Creative Commons Atribución 4.0 Internacional (CC BY 4.0)

Revista Cátedra, 3(2), pp. 35-51, mayo-agosto 2020. e-ISSN: 2631-2875

https://doi.org/10.29166/10.29166/catedra.v3i2.2188 
refiere a un modelo educativo unidireccional, puede diferir en imaginarios sociales distintos y con ello ajustarse a diferentes tipos de intereses.

Entre los principales propósitos de la educación intercultural en México se ha puesto énfasis en el auxilio a los estilos de vida y prácticas tradicionales en riesgo, como sucede con las prácticas de los grupos que se sostienen en torno a sus lenguas originarias. Sin embargo, como suele suceder cuando el discurso rebasa la realidad, la incorporación de la educación intercultural en México se ha distinguido por asumir una orientación integradora, de manera que ha buscado elevar las competencias de la población, en riesgo, a fin de que su participación al entorno nacional e internacional se realice en las mejores condiciones; pero carece de un sentido de pleno respecto para no dañar las condiciones originarias.

Con 20 años de existencia del modelo de Universidad Intercultural no se puede hablar de un fracaso, pero sí es posible mencionar que su propósito fundamental se encuentra estancado dentro de un ámbito político que ha permitido a los encargados de la construcción de la política educativa obtener una salida discursiva ante las demandas de los grupos, anteriormente, excluidos.

En términos cuantitativos, el modelo de la Universidad Intercultural en México no ha tenido el impacto esperado en su alcance de cobertura. Así, por ejemplo, en Aguirre (2015) se indicó que en el año 2015 las Universidades Interculturales en su conjunto atendieron una población que representó cerca del $18 \%$ de la población objetivo y apenas un $4.3 \%$ de la potencial; aunado a ello, estas universidades enfrentan un severo problema de deserción y de egresados no titulados, por lo que en lo general se menciona que ha sido un modelo cuya percepción es de inutilidad dentro de la educación formal.

\section{Aspectos metodológicos}

Este trabajo consideró el caso de dos universidades interculturales mexicanas seleccionadas por su representatividad respecto a la trayectoria histórica dentro del modelo de educación intercultural. Primero, la Universidad Intercultural del Estado de México cuya oferta académica recae en seis programas de estudio: Comunicación Intercultural, Lengua y Cultura, Desarrollo Sustentable, Salud Intercultural, Arte y Diseño y Enfermería (UIEM, 2019). Segundo, la Universidad Intercultural de Chiapas donde existen seis carreras del nivel superior: Comunicación intercultural, Lengua y cultura, Turismo alternativo, Desarrollo sustentable, Derecho intercultural, Médico cirujano (UNICH, 2018).

Puesto que la intención fue estudiar la percepción que se tiene por parte de los participantes del modelo sobre su desempeño como egresados, o profesionistas, interculturales, se propuso desarrollar un trabajo de tipo cualitativo que diera cabida a la recolección de la voz y opinión en dos dimensiones. Primero, la dimensión individual, en referencia a la relación entre la identidad cultural y la práctica profesional, ¿cómo se identifica y se valora el egresado intercultural? Segundo, la dimensión comunitaria, para evaluar la capacidad de la educación intercultural en la promoción del desarrollo de las comunidades de origen.

La primera fase de la investigación se realizó mediante la consulta de fuentes oficiales localizadas a través de la Asociación Nacional de Universidades e Instituciones de Educación Superior (ANUIES), y otros materiales emitidos por la SEP; esta parte fue complementada mediante consultas directas con el personal especializado en el seguimiento de egresados de ambas universidades seleccionadas. Con ello, se tuvo un acercamiento a la evolución en números de dichas universidades, como fue el grado de representatividad en referencia a la

Licencia Creative Commons Atribución 4.0 Internacional (CC BY 4.0)

Revista Cátedra, 3(2), pp. 35-51, mayo-agosto 2020. e-ISSN: 2631-2875

https://doi.org/10.29166/10.29166/catedra.v3i2.2188 
educación superior total en México, también se recuperó información sobre la matrícula de ingreso y egreso, y se realizó un rastreo de la colocación laboral de los egresados.

La segunda parte de la metodología fue de tipo cualitativa y se realizó mediante una recolección de datos bajo la técnica de la entrevista semidirigida, con una muestra estructurada que permitió acceder a los testimonios en dos niveles: el institucional, en apego a la opinión de las autoridades de los planteles seleccionados, y con egresados. Se reunieron 21 testimonios equitativamente divididos entre la UNICH y la UIEM, de las licenciaturas Turismo Alternativo (33.3 \%), Comunicación Intercultural (52 \%), Desarrollo Sustentable (9.52\%) y Lengua y Cultura ( $4.7 \%)$.

Para los egresados se determinó una muestra aleatoria, sin distinción respecto a la licenciatura de egreso, género u ocupación vigente. La muestra ubicó por sí misma la condición de los informantes en cuanto a su procedencia desde alguna comunidad indígena y su conexión con alguna lengua también originaria. En la muestra de la UNICH se observa mayor presencia de egresados con procedencia desde alguna comunidad rural o indígena; al mismo tiempo que se identifica la fuerte presencia de la lengua tzotzil, ya sea como primera lengua o como lengua de tradición dentro de las familias.

Para el caso de la UIEM la presencia en el manejo de alguna lengua originaria fue nula como primera lengua, aunque destacan las lenguas mazahua y otomí en el orden de uso de tradición familiar. En el Cuadro 1 se muestran las características de los egresados entrevistados.

\begin{tabular}{|c|c|c|c|}
\hline Entrevistado & Licenciatura & Procedencia & $\begin{array}{c}\text { Condición } \\
\text { cultural y } \\
\text { lingüística * }\end{array}$ \\
\hline \multicolumn{4}{|l|}{ UNICH } \\
\hline Teresa & $\begin{array}{l}\text { Turismo } \\
\text { Alternativo }\end{array}$ & $\begin{array}{l}\text { San Cristóbal de las } \\
\text { Casas }\end{array}$ & $\mathrm{TLI}^{2}$ \\
\hline Gloria & $\begin{array}{l}\text { Turismo } \\
\text { Alternativo }\end{array}$ & $\begin{array}{l}\text { San Cristóbal de las } \\
\text { Casas }\end{array}$ & Mestiza \\
\hline Diana & $\begin{array}{l}\text { Turismo } \\
\text { Alternativo }\end{array}$ & Pichucalco & Mestiza \\
\hline Enoc & $\begin{array}{l}\text { Turismo } \\
\text { Alternativo }\end{array}$ & La Frailesca & Mestizo \\
\hline Santos & $\begin{array}{l}\text { Turismo } \\
\text { Alternativo }\end{array}$ & San Pedro, Huixtan & $\mathrm{HLI}^{3}$ tzotzil \\
\hline Mario & $\begin{array}{l}\text { Turismo } \\
\text { Alternativo }\end{array}$ & San Marcos Tulijá & HLI tzeltal \\
\hline Juan & $\begin{array}{l}\text { Turismo } \\
\text { Alternativo }\end{array}$ & $\begin{array}{l}\text { Santa Martha, } \\
\text { Chenalhó }\end{array}$ & HLI tzotzil \\
\hline Sandra & $\begin{array}{l}\text { Comunicación } \\
\text { Intercultural }\end{array}$ & Ocosingo & TLI \\
\hline Abelardo & $\begin{array}{l}\text { Comunicación } \\
\text { Intercultural }\end{array}$ & $\begin{array}{l}\text { Amatenango de la } \\
\text { Frontera }\end{array}$ & TLI \\
\hline
\end{tabular}

\footnotetext{
2 TLI: persona con tradición lingüística indígena en la familia cuya primera lengua es castellano, pero, además, puede manejar en distintos grados la lengua originaria respectiva; mestizo: persona con el castellano como primera lengua, sin noción de raíces indígenas en la familia.

${ }^{3} \mathrm{HLI}$ : hablante de lengua indígena como primera lengua.
} 


\begin{tabular}{|c|c|c|c|}
\hline Miguel & $\begin{array}{l}\text { Comunicación } \\
\text { Intercultural }\end{array}$ & Carmito, Huitiupán & HLI tzotzil \\
\hline Francisco & $\begin{array}{l}\text { Comunicación } \\
\text { Intercultural }\end{array}$ & $\begin{array}{l}\text { San Martín, } \\
\text { Pantelhó }\end{array}$ & HLI tzotzil \\
\hline \multicolumn{4}{|l|}{ UIEM } \\
\hline Edén & $\begin{array}{l}\text { Comunicación } \\
\text { Intercultural }\end{array}$ & $\begin{array}{l}\text { Emilio Portes Gil, } \\
\text { San Felipe del } \\
\text { Progreso }\end{array}$ & TLI mazahua \\
\hline Ismael & $\begin{array}{l}\text { Comunicación } \\
\text { Intercultural }\end{array}$ & $\begin{array}{l}\text { San Felipe del } \\
\text { Progreso }\end{array}$ & Mestizo \\
\hline Norma & $\begin{array}{l}\text { Comunicación } \\
\text { Intercultural }\end{array}$ & Atlacomulco & TLI mazahua \\
\hline Yeni & $\begin{array}{l}\text { Comunicación } \\
\text { Intercultural }\end{array}$ & Jocotitlán & Mestiza \\
\hline Rosario & $\begin{array}{l}\text { Comunicación } \\
\text { Intercultural }\end{array}$ & $\begin{array}{l}\text { San Felipe del } \\
\text { Progreso }\end{array}$ & TLI mazahua \\
\hline Gaba & $\begin{array}{l}\text { Comunicación } \\
\text { Intercultural }\end{array}$ & $\begin{array}{l}\text { San Pedro de los } \\
\text { Baños, } \\
\text { Ixtlahuaca }\end{array}$ & TLI mazahua \\
\hline Hermilo & $\begin{array}{l}\text { Comunicación } \\
\text { Intercultural }\end{array}$ & $\begin{array}{l}\text { Álamo, Veracruz } \\
\text { (vive en Edomex) }\end{array}$ & Mestizo \\
\hline Alma & $\begin{array}{l}\text { Desarrollo } \\
\text { Sustentable }\end{array}$ & $\begin{array}{l}\text { Rincón de la } \\
\text { Candelaria, } \\
\text { Atlacomulco }\end{array}$ & Mestiza \\
\hline Anayeli & $\begin{array}{l}\text { Desarrollo } \\
\text { Sustentable }\end{array}$ & San José del Rincón & TLI mazahua \\
\hline Griselda & Lengua y Cultura & $\begin{array}{l}\text { Santa María } \\
\text { Nativitas, } \\
\text { Jiquipilco }\end{array}$ & TLI otomí \\
\hline
\end{tabular}

Cuadro 1. Egresados entrevistados y sus características personales

Con la información obtenida se trabajó en dos aspectos. Se preparó un indicador para evaluar la relación de congruencia entre el perfil de egreso (PE) y el tipo de trabajo (T) en el que se desempeñaba la persona al momento de la entrevista. Para estimar el grado de la congruencia entre el perfil y el trabajo (PE/T), se consideró como el primer momento distintivo de cada posición laboral el contacto con las comunidades (rurales y/o indígenas) mantenido por cada egresado, dado que el desempeño profesional en contextos comunitarios es la base misma del planteamiento de las licenciaturas interculturales.

Un segundo aspecto fue captar la percepción que cada uno de que los entrevistados posee sobre la relevancia por haber adquirido un perfil intercultural, junto a identificar, 0 comprobar, la relación existente entre su condición laboral en atención a las comunidades de origen. Adicionalmente, se tomó en cuenta si el egresado pone en práctica las habilidades adquiridas durante la licenciatura y si es consciente de que su formación universitaria fue diferente a la convencional. 


\section{Resultados}

El éxito de cada universidad intercultural puede medirse en el desempeño de sus egresados, o sea, mediante la congruencia entre la característica del desempeño laboral y el perfil de egreso correspondiente. El cuestionamiento inicial es si el potencial del modelo educativo de la Universidad Intercultural ha sido capaz de plasmarse en una formación sólida de egresados "de otro tipo" que consecuentemente actúen en la sociedad según los valores interculturales, algunos especialistas se muestran optimistas, por ejemplo, Sartorello 2007.

¿Qué sucede una vez concluida la formación universitaria intercultural? Cuantificar la colocación de los egresados en el mercado laboral y su éxito profesional no es nada nuevo en ninguna institución de educación superior. Sin embargo, en el caso de las universidades interculturales, el tema es más delicado que en otras partes debido al tipo especial de formación y función social que a ésta corresponde.

En el marco del discurso interculturalista, para los egresados se plantean metas muy ambiciosas, nada menos que crear espacios nuevos en el mercado laboral existente, saturado con los profesionistas "convencionales", valiéndose de una formación especialmente diseñada para traer ventaja comparativa a los jóvenes indígenas (González Ortiz, 2007a, p. 80). Y como señala Sartorello:

\section{Me atrevo a sugerir que estos jóvenes se están formando para ser agentes de cambio de sus pueblos y comunidades para contribuir a superar la marginación económica, social y política que padece su gente y, al mismo tiempo, fortalecer y revalorar la cultura propia como elemento aglutinador y creador de identidades étnicas en grado de resistir a las embestidas de la cultura occidental urbana (Sartorello, 2007, pág. 4).}

Pero ¿cuántos egresados realmente han logrado establecerse en áreas profesionales que están en sintonía con su formación intercultural? ¿Qué tan probable es que los exalumnos (sobre todo los que declaran tener una vocación "intercultural") podrán practicar plenamente dicha vocación fuera de su alma mater ante lo que Sartorello (2007) llamó las "embestidas de la cultura occidental urbana" (pág. 4) Tanto entre los egresados como entre los trabajadores institucionales existe la conciencia de que en las licenciaturas interculturales deben formarse profesionistas diferentes a los "convencionales"? Lo que falta es el consenso sobre si los egresados de carne y hueso realmente cumplen con esta característica. Primero se debe revisar la visión institucional compartida por los rectores de ambas Universidades respecto al desempeño de sus egresados.

El discurso de Javier Álvarez Ramos, rector de la UNICH entre 2011 y 2014, se caracteriza por dos posiciones en cierta medida incompatibles: conciencia sobre la misión de la Universidad Intercultural que debe formar a profesionistas "alternativos" para el trabajo comunitario (limitada por la realidad en que pocos exalumnos llegan a desempeñarse de este modo por buscar empleos más bien convencionales), y la tendencia a medir el éxito según los parámetros ligados a la competitividad, buenos puestos y elevados sueldos.

Diría que nuestros egresados tienen un 30\% de colocación inmediata. Desafortunadamente, no en la lógica del perfil de egreso de que regresen a su comunidad. Aquí chocan dos pretensiones y una es de índole cultural. En las comunidades indígenas, salir de la comunidad es motivo de progreso. Retornar es motivo de fracaso. Entonces encontramos una resistencia natural del chico para regresar a su comunidad y nuestros chicos buscan empleo en el mercado formal. No es ésa la pretensión de la

Licencia Creative Commons Atribución 4.0 Internacional (CC BY 4.0)

Revista Cátedra, 3(2), pp. 35-51, mayo-agosto 2020. e-ISSN: 2631-2875

https://doi.org/10.29166/10.29166/catedra.v3i2.2188 
Universidad. Se les prepara que sean capaces de gestionar proyectos y desarrollar gestión de dependencias fuera de ellas y armar proyectos culturales, productivos, desde y con la comunidad (Álvarez-Ramos, comunicación personal, 10-04-2014).

Más adelante se nota la complacencia del rector ante los casos de jóvenes con colocación empresarial, bien establecidos, subrayando el caso de un egresado gerente de marketing de los autos de la marca Nissan que atiende a la clientela indígena en su lengua materna. Aunque el rector es consciente de que no es el perfil deseable en que tal persona deba trabajar, no puede abstenerse de un tono entusiasmado ante tal "caso de éxito".

Tenemos ya dos empresas de jóvenes estudiantes que son gestores que cumplen perfectamente con la misión de la Universidad y con bastante éxito. Tenemos chicos, además, que han puesto su propia empresita, hay alguien quien tiene su restaurán (sic) por allí muy exitoso y produce sus alimentos y los hace allí. También tenemos en el empleo formal el gerente de marketing de Nissan San Cristóbal; me queda muy claro que no es su perfil, pero, ¿por qué lo contrata la Nissan? El principal consumidor de la camioneta nissancita aquí en Chiapas es el indígena y las ventas incrementaron porque el gerente habla en la lengua indígena con el comprador. Entonces hay casos de éxito, pero sigue siendo un reto para nosotros que la mayoría de nuestros chicos cumplan con la misión que nos tiene encomendada para efectos de su perfil de egreso (ÁlvarezRamos, comunicación personal,10-04-2014).

Por su parte, Monroy-Gaytán, rector de la UIEM, al momento de la entrevista, en su discurso calificó el desempeño de los egresados como más bien satisfactorio, aunque se señaló la necesidad de reciclar carreras más a menudo para evitar que el mercado regional se saturara.

Hay muchos (alumnos) de Lengua y Cultura que han salido al lado de la docencia, cosa que me parece pertinente porque hay docentes que no hablan las lenguas (originarias), entonces dedicarse a la docencia con este valor es positivo. (La carrera de) Desarrollo Sustentable se ha ido mucho a las organizaciones no gubernamentales y autogestionadas, haciendo proyectos en las comunidades (indígenas). Y Comunicación Intercultural ha estado vinculada en todos los medios regionales y locales, como las radios. Por el tamaño de la zona debe haber un proceso más rápido de reciclar carreras y cambiar sus perfiles. La Universidad Intercultural está pasando por una etapa de reflexión, de reestructuración, y de darse cuenta de cuáles son sus perfiles. Por ejemplo, la carrera de Salud Intercultural: no entendíamos bien cómo está el mercado, cuál debía de ser el perfil. Hoy lo tenemos claro: con una clínica, con un área de laboratorios trabajando fitofarmacia, ya entendemos cuál es nuestro lado más fuerte (Monroy-Gaytán, comunicación personal, 08-05-2014).

El directivo acentuó que la Universidad Intercultural se encontraba en proceso de autodefinición y que los perfiles de sus licenciaturas estaban apenas cristalizando. Este último argumento aparece también en el discurso de Felipe Medina, responsable del seguimiento de egresados de la UIEM, quien resumió las complicaciones enfrentadas por 
los exalumnos. El académico considera como exitosos a los egresados que sí supieron desenvolverse dentro de sus perfiles que implican el trabajo comunitario.

Somos una universidad de reciente creación y hay que consolidar imagen, entonces nuestros egresados tienen serios problemas de mil tipos, como la titulación, cursos de actualización, o que los reconozcan en otras instituciones. Tenemos una eficiencia terminal muy baja, tal vez el $60 \%$ más o menos, en titulación un $20 \%$. Hay mucho desempleo, desafortunadamente. Nos falta hacer mucho trabajo en cuestión al egresado emprendedor. De hecho, ése es el ideal de las Universidades Interculturales que se van ubicando en espacios de alta marginación, que los egresados hagan proyectos de desarrollo en sus mismas comunidades y que hagan impacto en esta marginación. Son pocos, realmente, los que han constituido asociaciones civiles que les permiten generar u obtener proyectos, y que a su vez tienen impacto en las comunidades. Son pocos, pero sí podemos mencionar algunos egresados de éxito (Monroy-Gaytán, comunicación personal, 08-05-2014).

Como se ejemplifica en los discursos reproducidos, y más allá, los problemas que enfrentan los egresados son múltiples. El modelo educativo de la Universidad Intercultural no cuenta con una difusión amplia, la cobertura en el marco de un país tan extenso como México es reducida, y la gran parte de la sociedad lo desconoce, lo que se convierte en un problema sobre todo cuando se trata de los empleadores. Además, el modelo está envuelto en un tejido de creencias, prejuicios, estereotipos y críticas en mayor o menor medida justificadas, que lo consideran como segregacionista, "para indios", "para pobres", de baja calidad académica o simplemente como utópico y, por ende, incompatible con la realidad globalizada del país.

Desde los archivos de las Universidades Interculturales fue posible obtener una serie de datos acerca de las condiciones laborales para los egresados de las mismas.

\begin{tabular}{|c|c|c|}
\hline Indicadores & UNICH & IUEM \\
\hline $\begin{array}{l}\text { Ámbito o tipo de desempeño } \\
\text { de los egresados empleados }\end{array}$ & $\begin{array}{l}83 \% \text { organizaciones o negocios } \\
8 \% \text { socios } \\
6 \% \text { dueños de empresas }\end{array}$ & $\begin{array}{l}93 \% \text { organizaciones } 0 \\
\text { negocios } \\
7 \% \text { dueños de empresas }\end{array}$ \\
\hline Tipo de contrato & $\begin{array}{l}35 \% \text { de tiempo indefinido } \\
54 \% \text { de tiempo definido } \\
11 \% \text { otro }\end{array}$ & $\begin{array}{l}54 \% \text { de tiempo indefinido } \\
33 \% \text { de tiempo definido } \\
13 \% \text { otro }\end{array}$ \\
\hline Nivel de ingreso (mensual) & $\begin{array}{l}25 \% \text { menos de } 2 \text { mil pesos } \\
48 \% 2 \text { a } 5 \text { mil pesos } \\
23 \% \text { más de } 5 \text { a } 10 \text { mil pesos } \\
3 \% \text { sin sueldo (parcela propia, } \\
\text { negocio familiar) }\end{array}$ & $\begin{array}{l}\text { Ingreso promedio neto de } \\
4575 \text { pesos }\end{array}$ \\
\hline $\begin{array}{l}\text { Desempeño basado en el } \\
\text { contacto con comunidades }\end{array}$ & $\begin{array}{l}45.5 \% \text { Sí } \\
54.5 \% \text { No }\end{array}$ & $\begin{array}{l}\text { 40\% Sí } \\
60 \% \text { No }\end{array}$ \\
\hline Razón del desempleo & $\begin{array}{l}78 \% \text { por bajos salarios } \\
14 \% \text { por estar pendientes de la } \\
\text { titulación }\end{array}$ & $\begin{array}{l}52 \% \text { por no encontrar } \\
\text { trabajo } \\
17 \% \text { por seguir estudiando }\end{array}$ \\
\hline
\end{tabular}

Cuadro 2. Situación laboral de los egresados de la UNICH y la UIEM. Fuente: Compilación propia con base en Hartmann y López (2013) y Medina y Hernández (2014)

Licencia Creative Commons Atribución 4.0 Internacional (CC BY 4.0)

Revista Cátedra, 3(2), pp. 35-51, mayo-agosto 2020. e-ISSN: 2631-2875

https://doi.org/10.29166/10.29166/catedra.v3i2.2188 
En el Cuadro 2 se observa que, en su mayoría, los egresados de las universidades interculturales dependen laboralmente de los puestos de trabajo de organizaciones o negocios ya establecidos; un porcentaje bajo atiende empresas o negocios propios. El tipo de contratación que han asumido mayoritariamente tiene una duración definida, o temporal, para los egresados de la UNICH y mayoritariamente de tipo indefinido para los egresados de la UIEM, aunque este indicador no es relevante para evaluar el desempeño de un profesionista intercultural, porque los tipos de contrato pueden asumirse como parte de la propia conformación del mercado laboral.

El ingreso salarial promedio reportado, en ambos casos, es bajo considerando que se trata de profesionistas. Este es un dato alarmante cuando se conocen los testimonios de los egresados, quienes indican la existencia de demerito para un egresado intercultural, como se constata en los comentarios de los egresados 4 .

A pesar de que el dato sobre los egresados empleados, o no, resultó ambiguo ha sido posible ubicar la problemática de dicho desempeño. Los egresados que manifestaron estar en condición de desempleo señalaron que son los bajos salarios la razón principal. En este sentido se ha formado un círculo vicioso, la situación para los estudiantes se agrava por la escasez de recursos económicos, ya sea para culminar la carrera o para pagar los derechos de titulación (sobresale el caso de los egresados no titulados de la UNICH: $65 \%$ ) lo cual repercute sobre la calidad de las opciones laborales, ya que sin título el joven no cobra un sueldo de profesionista sino de técnico.

\begin{tabular}{lcc}
\hline Indicadores & UNICH & IUEM \\
\hline Trabajan dentro del perfil & $34 \%$ & $33 \%$ \\
$\begin{array}{l}\text { Su trabajo tiene algo o poco que } \\
\text { ver con el perfil }\end{array}$ & $60.6 \%$ & $19 \%$ \\
$\begin{array}{l}\text { Su trabajo no se relaciona con el } \\
\text { perfil }\end{array}$ & $5.4 \%$ & $39 \%$ \\
\hline
\end{tabular}

Cuadro 3. Congruencia entre el perfil de egreso y el trabajo (CE/T). Fuente: Compilación propia con base en Medina y Hernández (2014, p. 37) y Hartmann y López (2013, p. 13).

Continuando con los datos extraídos desde las instituciones, en el Cuadro 3 se presenta la relación de congruencia entre el perfil de egreso y empleo actual; en este campo existe semejanza, entre ambas Universidades, pues los egresados registrados que trabajan dentro del perfil para el cual fueron de formados son: $34 \%$ para la UNICH, y $33 \%$ para la UIEM. Sin embargo, para la UIEM se muestra mayor problema respecto al conglomerado de egresados que declaran atender ámbitos escasamente, o nulamente, relacionados con su perfil $39 \%$.

Ahora bien, las experiencias personales, opiniones y trozos significativos de narrativas vitales de los exalumnos abrieron la puerta hacia la actuación social (tanto a su concepción intelectual como a las prácticas reales) de este nuevo tipo de profesionista. A continuación

${ }^{4}$ Los estados de México y de Chiapas se ubican dentro del grupo de las 10 entidades con el menor nivel de ingreso promedio, por debajo de la media nacional (que corresponde a los 6,405 pesos mensuales, alrededor de los 330 dólares por mes; STPS, 2020). 
se mostrará la percepción que se tiene sobre la formación intercultural por parte de los egresados.

Respecto a los motivos para ingresar en los estudios interculturales se tienen diferencias entre los estudiantes de la UNICH y la UIEM. Al parecer, los jóvenes postulantes en Chiapas poseen mayor conciencia sobre el modelo intercultural. Al respecto, entre los principales motivos que condicionan su ingreso se detectaron: la cercanía y el interés por las lenguas originarias, seguido de factores económicos junto con identificar un modelo innovador propuesto con carreras alternativas que no podrían ser adquiridas en otros modelos. En cambio, en la UIEM, los postulantes declaran que las dificultades económicas y la cercanía a la Universidad fueron los principales motivos para su elección, seguido de los factores que la interpretan como un modelo innovador. También hay un sector de estudiantes que realizó comentarios no favorables para la educación intercultural al señalar que fue su última opción cuando en realidad buscaban participar en otros modelos educativos, estos egresados interpretan que el modelo no funciona como se expone en sus bases.

Escasamente, la percepción de los entrevistados fue haber ingresado a un modelo educativo que les permitiría posicionarse dentro del mercado de trabajo de manera plena y con ventajas. En su mayoría, señalan que la parte favorable de la educación intercultural fue el cambio actitudinal que experimentaron a lo largo de la carrera. Se debió a que percibieron una transformación hacia la valoración del modelo y las ventajas que puede arrojar, aunque no relacionadas con el mercado laboral sino con una posición más bien de enfoque social, y hasta altruista. Lo anterior les permite entenderse como potenciales promotores de cambio y desarrollo en sus comunidades de origen.

En cuanto al grado en que los egresados aplican la misión y visión de la Universidad Intercultural en sus lugares de trabajo se tiene que el 36,4\% de los egresados de la UNICH afirma que la visión intercultural universitaria no sólo transformó su manera de pensar, sino que, además, se proyecta en su manera de trabajar. El resto se muestra menos optimista: hay quienes simplemente no tienen empleo por el momento (36.4\%) y otros trabajan en ámbitos donde no se promueve el diálogo intercultural y sus propuestas no se toman en cuenta (27.3 \%) (Hartmann y López, 2013, p. 16). Es decir, la misión de la Universidad Intercultural no se cuestiona demasiado, pero la situación imperante en la sociedad y específicamente en el mercado laboral impide convertirla en una práctica real. Los exalumnos de la UNICH recomiendan que la educación intercultural debe ampliar su difusión: como institución (43\%) y ampliar su labor (19\%), además de contratar a docentes más capacitados (26 \%) (Hartmann y López, 2013, p. 17).

No existen datos comparables sobre la UIEM, pero según Medina-Flores y Hernández-García más de la mitad de los egresados está muy satisfecha o satisfecha con su papel de hacer algo útil por la sociedad (20\% y $43 \%$, respectivamente) y el $15 \%$ y $57 \%$ dijeron estar muy satisfechos o satisfechos con la puesta en práctica de los conocimientos adquiridos en la UIEM. Esto deja a la UIEM en mejor luz que la UNICH, o tal vez refleja una actitud menos crítica de los egresados mexiquenses frente a los chiapanecos (Medina-Flores y Hernández2014, pp. 28,49) ¿Cómo es la situación particular de nuestros informantes y hasta dónde les fue posible mantenerse dentro de sus perfiles profesionales?

Resulta que seis egresados (tres de la UNICH y tres de la UIEM) tienen la relación de congruencia PE/T más alta posible, ya que manifiestan perfiles laborales positivos. Pero también hay numerosos casos de egresados quienes trabajan en ámbitos comunitarios y utilizan los conocimientos aprendidos en la Universidad Intercultural, aunque personalmente no sientan gran influencia de la formación intercultural recibida (en total son nueve; cinco en la UNICH y cuatro en la UIEM). También hay quienes por el momento

Licencia Creative Commons Atribución 4.0 Internacional (CC BY 4.0)

Revista Cátedra, 3(2), pp. 35-51, mayo-agosto 2020. e-ISSN: 2631-2875

https://doi.org/10.29166/10.29166/catedra.v3i2.2188 
no se mueven en los ámbitos comunitarios y no practican sus competencias interculturales, pero esperan hacerlo pronto: se entrevistó a cuatro egresados chiapanecos que son empleados de alguna organización, asociación civil, ONG, etc., y que estaban esperando a que les llegara la oportunidad de volver a hacer el trabajo de campo, aunque sus puestos en aquel momento no lo requerían. En cambio, entre los egresados del estado de México prevalecen quienes sí expresaron el deseo de hacer el trabajo de campo, pero debido al carácter de sus puestos laborales no veían muy probable que algún día sucediera (cinco personas de la UIEM).

También hay casos donde la PE/T es baja o de plano ninguna. La situación para el egresado es la siguiente: a) no trabaja dentro de su perfil y a esto se suma su enajenación laboral respecto al ámbito comunitario (tres casos en la UIEM y ninguno en la UNICH); b) trabaja dentro de su perfil pero sin estar en contacto con las comunidades (dos casos en la UNICH); c) trabaja dentro de su perfil, pero sin mantener el enfoque intercultural y sin desempeñarse en el ámbito comunitario (dos casos en la UIEM).

En los casos b) y c) se trata de los egresados de la Comunicación Intercultural y los distingue una circunstancia. En el caso b), los dos egresados trabajan en el ámbito de una Universidad Intercultural, es decir, atienden la problemática de la diversidad cultural en sus trabajos de comunicólogos, aunque no se requiere que hagan trabajo de campo. En el caso c) no existe tal enfoque, ya que el primer egresado trabajaba en el área de comunicación de la policía y el segundo para una radiodifusora privada.

En resumen, todos los 11 egresados chiapanecos trabajaban en menor o mayor medida dentro de sus perfiles profesionales y aproximadamente la mitad de ellos también se dedicaba al trabajo de campo. En cambio, sólo 4 de los 10 egresados mexiquenses trabajaban claramente de acuerdo con sus perfiles y al mismo tiempo se movían en ámbitos comunitarios.

Aquí entran las diferencias regionales que probablemente tienen que ver con el diferente grado de cohesión y vitalidad de las estructuras socioculturales originarias en ambas regiones. Este contexto proporciona más puestos laborales de tipo "intercultural" a los egresados chiapanecos que a los mexiquenses que habitan una zona mayoritariamente urbanizada e industrializada. Sin embargo, la UNICH tampoco está exenta del problema: los egresados entrevistados de ambas Universidades Interculturales frecuentemente ponían en tela de juicio la utilidad de la formación intercultural en el mercado existente y señalaban la dificultad de crear un nuevo espacio laboral para ellos mismos. Aun así, en muchos casos, su vocación por dedicarse a ocupaciones de tipo "intercultural" era fuerte, aunque por el momento no podía convertirse en prácticas reales.

Sin embargo, acorde con la competitividad que pudiera denotarse en relación con otros modelos educativos, la Universidad Intercultural orienta un desempeño más bien altruista, con capacidad para generar la promoción para el cambio de sus ambientes de origen. Y es en este punto donde hay controversia cuando el egresado se valora en función de sus oportunidades individuales para dirigirse al desempeño laboral. De hecho, hay comentarios entre los egresados que aseguran que el modelo dista de sus logros propuestos al identificarlo como no funcional. La aspiración máxima que se percibe es lograr ocupar cargos en la docencia o en la investigación social.

Licencia Creative Commons Atribución 4.0 Internacional (CC BY 4.0)

Revista Cátedra, 3(2), pp. 35-51, mayo-agosto 2020. e-ISSN: 2631-2875

https://doi.org/10.29166/10.29166/catedra.v3i2.2188 


\section{Conclusión}

La Universidad Intercultural se percibe como un modelo alternativo que oferta oportunidades a jóvenes que provienen sobre todo de contextos rurales o comunitarios, y que de otra manera no dispondrían de las posibilidades de acceso a los estudios universitarios en otras partes. El propósito de los estudios superiores interculturales observado en sus diferentes componentes (perfil de ingreso, perfil de egreso, misión y visión) es, en efecto, crear un tipo de profesionista diferenciado por el hecho de ser capaz de atender los problemas que se suscitan en el marco de la pluriculturalidad. Sin embargo, en la práctica real dentro de la sociedad y el mercado de trabajo, los egresados no ubican la manera clara de insertarse en las condiciones ideales para las que fueron formados.

Hay una versión oficial que no oculta los problemas a los que se están enfrentando los jóvenes egresados, pero se percibe un discurso de confianza al suponer que lentamente se abrirán los espacios laborales para que los egresados logren convertirse en promotores de desarrollo para los contextos locales y regionales. Adicionalmente, quienes dirigen estas instituciones enfatizan que ante las condiciones económicas desfavorables de las que provienen sus estudiantes, la educación universitaria intercultural permite equilibrar, en alguna medida, esta situación de desigualdad. En su mayoría los jóvenes tanto en la UNICH como en la UIEM provienen de familias que carecen de ingresos suficientes para permitir altos costos de matriculación, quizás pagos de colegiatura, traslados, hospedajes, etc., ante lo cual el modelo intercultural resulta una oportunidad única.

Finalmente, la percepción generalizada que sostuvieron los entrevistados, indistintamente de la UNICH o de la UIEM, respecto a la relación existente entre el perfil de egreso y los principios de la interculturalidad es de tipo positivo. Los egresados coinciden en que las carreras en las que participaron les ha permitido desarrollar un tipo de trabajo alternativo e innovador que difiere de la educación convencional por la capacidad de acercamiento a los espacios comunitarios, adicionalmente se perciben como entes generadores de cambio, personas con una mayor sensibilidad y conciencia que enfatiza en los grupos indígenas.

A pesar de lo anterior, los egresados hacen saber, mediante sus testimonios, que en el ámbito laboral permea el desconocimiento y la discriminación por la educación intercultural; se han dado cuenta que el nivel salarial tiende a ser más bajo para ellos que el que se otorga a egresados de otros ámbitos educativos. Las posibilidades de desarrollo de sus profesiones no están insertadas en programas públicos o privados que alienten su incorporación eficaz.

\section{Agradecimientos}

Dirigimos un agradecimiento a todos quienes colaboraron para dar sentido al proyecto de investigación, a quienes vertieron su opinión: egresados, autoridades, personal administrativo.

\section{Bibliografía}

Aguirre, M. M. (2015). Comunidades de aprendizaje: retos para la ampliación del impacto social de las Universidades Interculturales. México: Documento de trabajo 186, LXII Cámara de diputados.

Cárdenas, E. (2014). Migración interna e indígena en México: enfoques y perspectivas. Intersticios Sociales, 0(7), 1-28. 
CDI. (2018). Medición de la pobtreza 2015 en los municipios con ploblación indígena. México: Comisión para el Desarrollo de los Pueblos Indígenas.

CONEVAL. (2018a). Población indígena con carencias en todos sus derechos sociales (comunicado de prensa). México: Consejo Nacional de Evaluación de la Política Social.

Erdösová, Z. (2013). La universidad intercultural latinoamericana como un fenómeno múltiple. Una aproximación desde la teoría de los modelos educativos ecuatoriano y mexicano. Cuadernos interculturales, 11(21), 59-84.

González Ortiz, F. (2007). Crítica de la interculturalidad: la construcción de un proceso en el marco de la modernización. Cuadernos interculturales, 5(9), 63-89.

Granados-Alcantar, José A. y Quezada-Ramírez María F. (2018). Tendencias de la migración interna de la población indígena en México 1990-2015. Estudios Demográficos y Urbanos, 33(2), 327-363.

Hartmann, A. y López M. (2013). Resultados de las encuestas aplicadas a la primera generación de egresados (dos años después de su egreso 2009). San Cristóbal de la Casas: UNICH.

INEE. (2017a). Directrices para mejorar la atención educativa de niñas, niños y adoscentes indígenas, Instituto Nacional para la Evaluación de la Educación, México. Ciudad de México: Fondo editorial INEE.

INEE. (2017b). Políticas para mejorar la atención educativa de niñas, niños y adoscentes indígenas, Instituto Nacional para la Evaluación de la Educación, México (Documento ejecutivo). Ciudad de México: Fondo editorial INEE.

INEGI. (2018). Encuesta Nacional de Ocupación y Empleo, Instituto Nacional de Estadística y Geografía, México. México: INEGI.

INEGI. (2020). Estadísticas a propósito del día internacional de la eliminación de la discriminación racial (comunidao de prensa), Instituto Nacional de Estadística y Geografía, México. México: INEGI.

INPI. (2010). Indicadores socioeconómicos de los pueblos indígenas, 2015. México: INPI.

Medina-Flores, F. y Hernández-García, R. (2014). Programa de seguimiento de egresados. San Felipe del Progreso: Universidad Intercultural del Estado de México

Sartorello, S. (2007). Conversaciones interculturales: L@s estudiantes de la primera generación de la UNICH hablan de su universidad. San Cristóbal de las Casas: Gaceta, UNACH.

SEP. (2018). Universidades interculturales. México: Coordinación General de Educación Intercultural y Bilingüe, SEP.

STPS. (2020). Información laboral, enero 2020. México: STPS.

UIEM. (2019). http://uiem.edu.mx/.

UNESCO. (2006). Directrices de la UNESCO sobre la educación intercultural. París: UNESCO.

UNICH. (2018). https://www.unich.edu.mx/.

Licencia Creative Commons Atribución 4.0 Internacional (CC BY 4.0)

Revista Cátedra, 3(2), pp. 35-51, mayo-agosto 2020. e-ISSN: 2631-2875

https://doi.org/10.29166/10.29166/catedra.v3i2.2188 
Vázquez-Parra, J. y Campos-Rivas, C. (2016). Discriminación laboral indígena: una aproximación desde el imaginario colonial y la teoría elsteriana. Saber, 28(4), 828837.

\section{Autores}

ZUZANA ERDÖSOVÁ es Doctora en Estudios Latinoamericanos por la Universidad Autónoma del Estado de México (2015, mención honorífica y presea Ignacio Manuel Altamirano) y Doctora en Lenguas Romances por la Universidad de Palacký en la República Checa (2012). Es Maestra y Licenciada en Humanidades por la misma máxima casa de estudios checa. Desde 2016 se desempeña como profesora investigadora de tiempo completo en el Centro de Investigación en Ciencias Sociales y Humanidades (CICSyH) de la UAEM.

Es ganadora de la XIVa edición del Premio Iberoamericano (República Checa, 2009), candidata a Investigadora Nacional (SNI) y cuenta con el perfil deseable PRODEP. Es líder del Cuerpo Académico "Sociedades y Culturas de América Latina" adscrito a la Secretaría de Educación Pública en México. Es autora y coautora de varias decenas de publicaciones científicas sobre temas relacionados con su línea de investigación "interculturalidad, educación y lenguaje en América Latina". Ha sido ponente en diversos foros científicos nacionales e internacionales y ha dirigido diferentes proyectos de investigación. Se ha desempeñado como traductora, intérprete y también promotora de la cooperación académica y cultural entre México y la República Checa.

RAFAEL JUÁREZ-TOLEDO obtuvo el grado de doctor en Ciencias Sociales por la Facultad de Ciencias Política y Sociales de la Universidad Autónoma del Estado de México en 2015. Es maestro y licenciado en Economía por la misma Universidad. Desde 1996 se desempeña como profesor investigador de la Facultad de Economía de la Universidad Autónoma del Estado de México. También se ha desempeñado como profesor por asignatura del Instituto Tecnológico de Toluca. Cuenta con el perfil deseable PRODEP. Pertenece al Cuerpo Académico: Economía, Educación y Sustentabilidad adscrito a la Secretaría de Educación Pública en México.

Sus principales líneas de investigación se relacionan con los estudios socioterritoriales, la exclusión social y la pobreza, la migración, la sustentabilidad y las nuevas vertientes de la economía, y los estudios de la complejidad. Ha participado en diferentes tipos de publicaciones: material didáctico, revistas y libros. 